\title{
Otto Staudinger (1867-1952) - Geschichtsforscher Löbaus
}

\author{
Gerhard Staudinger
}

Als im April des Jahres 1900 der Seminaroberlehrer und angehende Fachlehrer für Französisch Otto Staudinger in Löbau eintraf, weil er vom Rochlitzer Lehrerseminar an das Löbauer Lehrerseminar versetzt wurde, hatte wohl niemand geahnt, dass soeben der künftige „beste Kenner der Löbauer Stadtgeschichte“ und spätere Löbauer Ehrenbürger „zugereist“ war.

Hermann Otto Staudinger wurde am 2. Dezember 1867 in Adorf im Vogtland als drittes Kind des Advokaten und Notars Julius Hermann Staudinger und dessen Frau Bertha Rosalie geb. Schneider geboren. Beide entstammten alteingesessenen und weitverzweigten vogtländischen Familien.

Ab 1873 besuchte Otto die Bürgerschule in Adorf, und es schien ausgemacht, dass er einst wie sein Vater und sein Großvater Jurist werden würde. Nun, daraus sollte nichts werden. Als der Vater viel zu früh und unerwartet 1878 starb, war Otto erst zehn Jahre alt. Die wirtschaftliche Situation der vaterlosen Familie war dadurch künftig angespannt und hatte Einfluss auf den weiteren Bildungsweg. Noch im gleichen Jahr zog die verwitwete Mutter mit ihren Kindern Marie und Otto nach Reichenbach im Vogtland, wo ein Teil ihrer Familie lebte. Ottos älterer Bruder Max war bereits als Kleinkind gestorben. Mit dem Umzug nach Reichenbach i.V. wechselte Otto Staudinger auf die Realschule, die er 1883 mit Bestnote abschloss. Trotzdem kam eine weitere akademische Ausbildung an einer Universität aus finanziellen Gründen nicht infrage, so dass die Mutter für ihren Sohn den Beruf des Volksschullehrers auswählte. ${ }^{1}$ Von 1883 bis 1887 absolvierte er das Lehrerseminar in Plauen.

Er begann anschließend seine berufliche Laufbahn als Hilfslehrer ${ }^{2}$ und nach bestandener „Wahlfähigkeitsprüfung“ ab 1890 als ständiger Lehrer ${ }^{3}$ jeweils an der I. Bürgerschule Reichenbach im Vogtland. Die Schwester hatte inzwischen geheiratet, während die Mutter den Haushalt des Sohnes versorgte.

Seine Zielstrebigkeit, die ihn lebenslang auszeichnete, führte $1895 \mathrm{zu}$ seiner Berufung als ständiger Lehrer an das neu gegründete Lehrerseminar in Rochlitz. Unterstützt wurde dieser Wechsel u. a. durch die Fürsprache seines frü- heren Direktors an der I. Bürgerschule Reichenbach im Vogtland, der inzwischen Bezirksschulinspektor in Löbau war.

Ostern 1895 trat Otto Staudinger sein Amt in Rochlitz an. Auch hier stürzte er sich mit Enthusiasmus an die Arbeit und wurde 1898 zum Oberlehrer ernannt. Zielstrebig vertiefte Otto Staudinger seine Französischkenntnisse. Nach Studien und Ferienkursen zur Erlernung der französischen Sprache an den Universitäten Genf, Paris, Marburg und Grenoble legte er im Herbst 1900 in Dresden die staatliche Fachlehrerprüfung für Französisch $a^{4}$. Aber auch danach belegte er noch weitere Kurse in Grenoble und Nancy. Erst aufgrund des Ersten Weltkriegs rissen die über 20 Jahre gepflegten Kontakte ab. Beim Singen in der „Liedertafel“ Rochlitz lernt Otto Staudinger seine spätere Frau Johanna, die älteste Tochter des Kaufmanns, Stadtrats und Landtagsabgeordneten Oscar Liebau ${ }^{5}$ und dessen Ehefrau Helene, geb. Schmieder, kennen. Weil im Seminar Löbau ein junger Lehrer plötzlich verstorben war, wurde Otto Staudinger kurzfristig ab Ostern 1900 nach Löbau versetzt, vielleicht abermals auf Empfehlung seines früheren Gönners, des Löbauer Bezirksschulinspektors.

Bevor er aus Rochlitz abreiste, verlobte er sich am 24. März 1900 mit Johanna Liebau, was zeitgemäß im Rochlitzer Amtsblatt angezeigt wurde. ${ }^{6}$ Am 22. Juli 1901 erfolgte die Trauung in der Rochlitzer Kirche St. Kunigunden. ${ }^{7}$ Da war Otto Staudinger bereits seit reichlich einem Jahr ein Löbauer Bürger. Das erhielt er bald darauf auch schriftlich, als ihm der Stadtrat zu Löbau mit Datum vom 24. Oktober 1902 einen „Bürgerschein“ ausstellt, wonach er „als Bürger der Vierstadt ${ }^{8}$ Löbau aufgenommen und verpflichtet worden ist".

Seine erste Wohnung in Löbau hatte er auf der Dammstraße 14, die heutige Breitscheidstraße 23, ehe er etwa 1903 eine neue Wohnung in der 1. Etage, Maschinenhausstraße 3 bezog, als die Familie Zuwachs bekam. Hier befand sich fortan das Zentrum der Familie Staudinger. Seine Frau wohnte dort bis zu ihrem Tod 1971. Das Wohnzimmerfenster gab den Blick auf die große Eisenbahnanlage mit Lokschuppen und Drehscheibe frei. Stundenlang haben dort spä- 


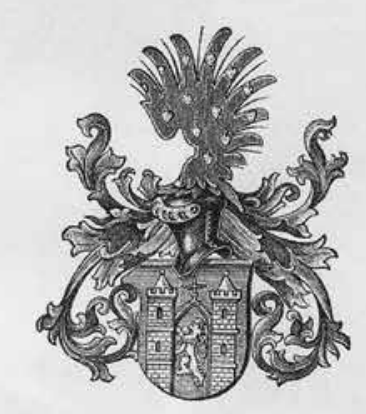

Bürgerschein der Stadt Löbau für Otto Staudinger

Aus: Rudolf Drescher: Löbau. Die

Stadt am Berge. Erfurt 1999, S. 20

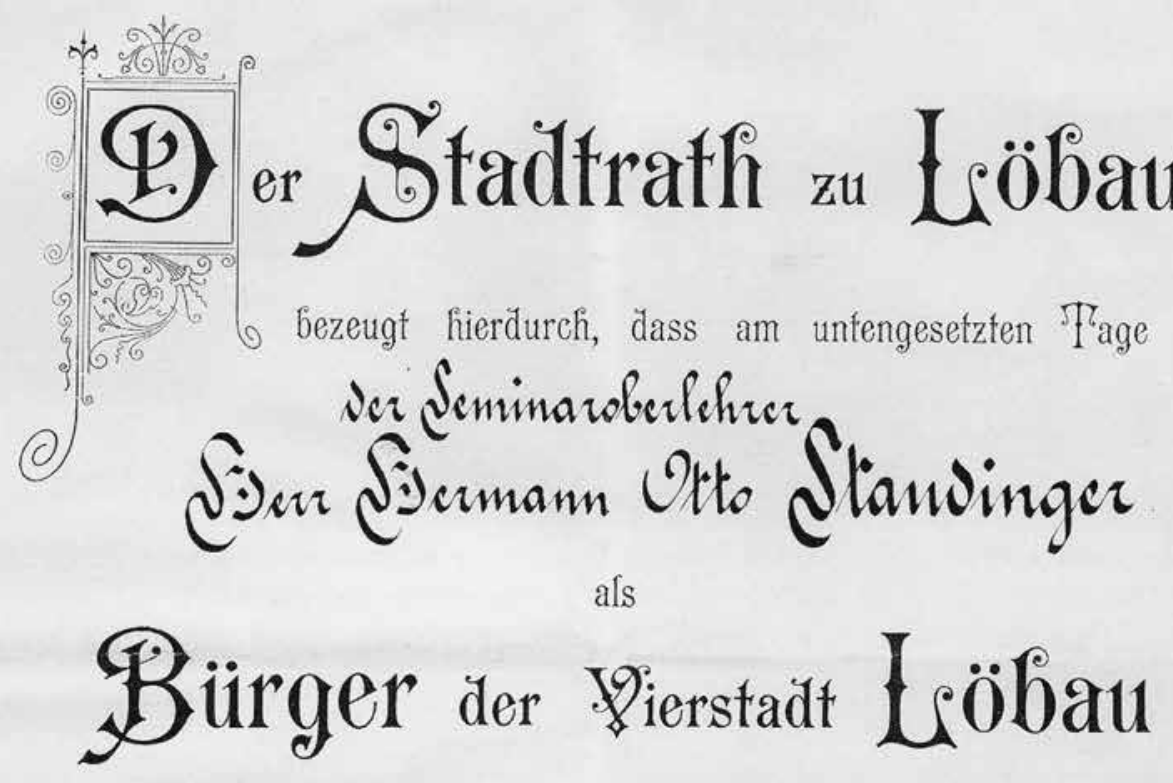

aufgenommen und verpflichtet worden ist.

Urkundich ist hierüber gegenwärtiger

\section{Bürgerschein}

ausgefertiget worden.

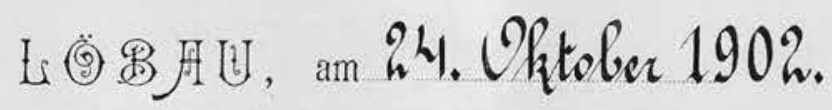

\section{Der Stadtrath daselbst.}

ter die Enkel beim Beobachten des regen Eisenbahnbetriebs die Zeit verbracht.

Auch seine Mutter hatte Otto Staudinger nach Löbau geholt, die alle die Jahre bis zu ihrem Tod 1920 im Alter von 87 Jahren bei der Familie ihres Sohnes in der Löbauer Wohnung wohnte. Für die junge Schwiegertochter war die Situation bestimmt nicht immer ganz einfach.

Zumal die Familie nun rasch anwuchs. 1903, 1906, 1908 und 1910 wurden die drei Töchter
Elisabeth, Elfriede und Gertrud sowie sein Sohn Eberhard geboren. 1917 folgte als Nachzügler der zweite Sohn Oskar. Die Töchter erlernten alle einen Beruf, zwei wurden Lehrerinnen, was in der damaligen Zeit nicht selbstverständlich war. Der älteste Sohn wurde Jurist, der jüngste Sohn fiel 1943 als Kapitänleutnant.

Am Löbauer Seminar auf der damaligen Seminarstraße 5, heute Pestalozzistraße 21, lehrte Otto Staudinger Deutsch, Mathematik, Turnen 


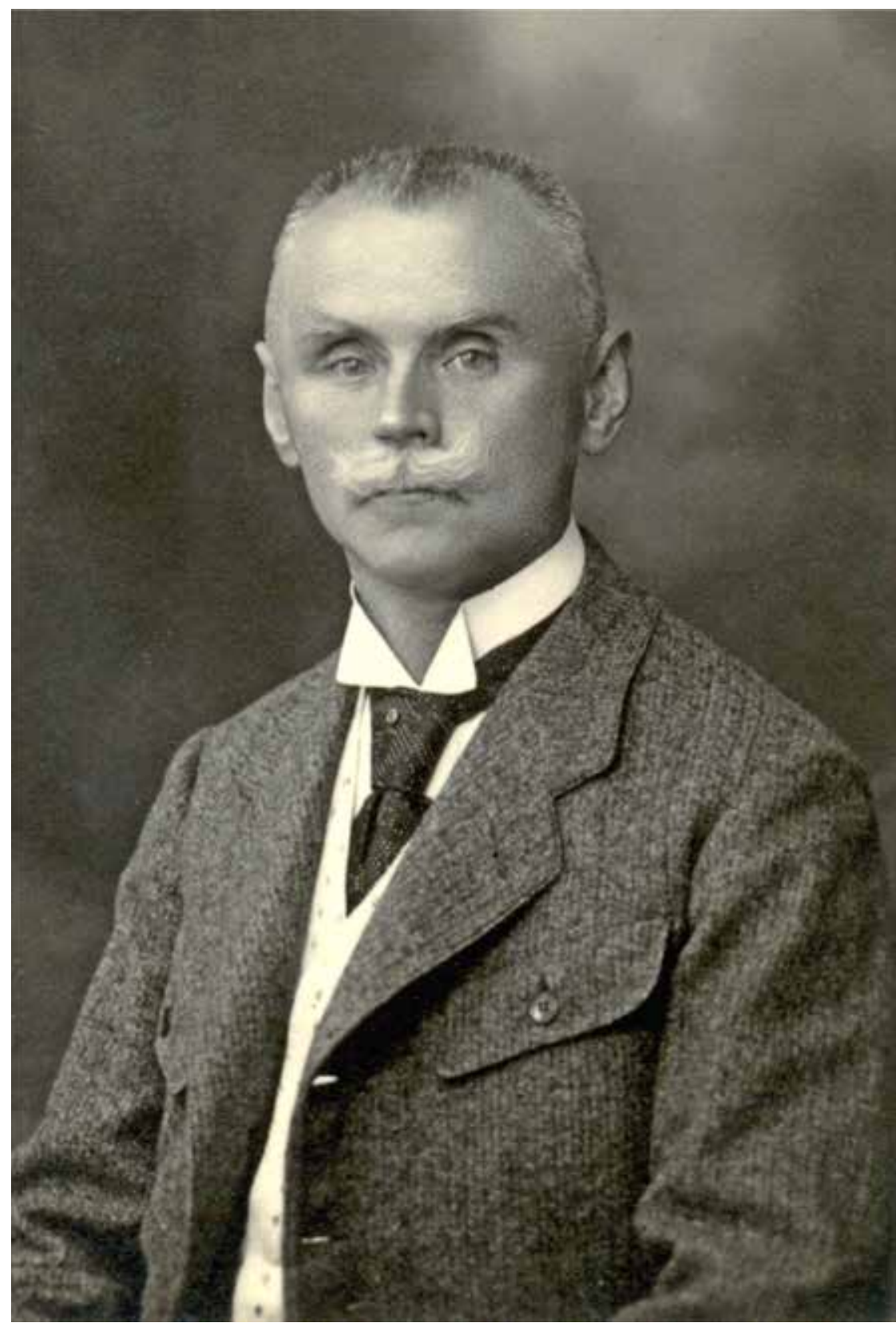

Otto Staudinger

9 Aufzeichnungen Otto Staudingers im Familienbesitz.

10 Abschrift der Rede vom 18. März 1932 im Familienbesitz.

11 Auszüge, Prof. Otto Staudinger betreffend, im Familienbesitz.

12 Görner: Biographischer Abriß von Prof. Otto Staudinger, in Veröffentlichungen des Stadtarchivs Löbau, 07/96, S. 4-5

13 Brief im Familienbesitz. und bevorzugt Französisch. Dabei hat er sehr begrüßt, dass beginnend ab Ostern 1904 der Französische Sprachunterricht zu Lasten von Latein aufgewertet wurde. Im Jahr 1920 erhielt Otto Staudinger den Titel eines Professors und 1921 Studienrat. ${ }^{9}$

Nach 32 Jahren am Seminar Löbau, das inzwischen zur Deutschen Oberschule umgewandelt worden war, und insgesamt 45 Jahren Arbeit im Lehrerberuf, trat Otto Staudinger am 31. März 1932 in den vorzeitigen Ruhestand. Er tat dies noch vor Erreichen der Altersgrenze und freiwillig, wie andere sächsische Lehrer damals auch, um jungen Lehrern Platz zu machen, die wegen schrumpfender höherer Schulen infolge der Weltwirtschaftskrise von Arbeitslosigkeit bedroht waren. Aus der Abschiedsrede des Rek- tors ${ }^{10}$ soll nur weniges zitiert werden: „In dieser schulpolitisch ... bewegten Zeit stand er immer mit an der Spitze der Vorwärtsschauenden und Vorwärtsstrebenden“. „Er war ein Lehrer, der mit weitem Blick die großen Fragen der Schularbeit und des Lehrerstandes umfasste und für jeden Schulfortschritt seine ganze Kraft einsetzte“.

Oskar Schwär (1890-1968) aus Dürrhennersdorf, Lehrer in Dresden und Heimatdichter der Oberlausitz, verfasste 1948 das (ungedruckt gebliebene) Manuskript zu seinem autobiographischen Buch „Sechs Jahre Kasten"11 in dem er seine Zeit im Löbauer Seminar 1904 bis 1910 schildert. Dort schreibt er: „Es bedeutete ein Glück für mich, daß Oberlehrer Staudinger (in der Quinta) mein Klassenlehrer wurde und bis ins letzte Jahr blieb“. Besonders hebt er dabei die Geduld bei der Vermittlung von Wissen und den guten Französischunterricht hervor und dass es ihnen „Stadl“ (wie er genannt wurde) bei den unvergesslichen Schulwanderungen „nicht gerade bequem“ machte.

Ja, der Staudinger-Geschwindschritt war Legende und auch gefürchtet. Die Oberlausitz, aber auch das Riesengebirge und die Alpen wurden erwandert, und Ehefrau Johanna mit den Kindern musste im vorgegebenen Tempo mit. Als Spaziergang bis ins hohe Alter wurde regelmäßig der Löbauer Berg erklommen.

1912 wurde Otto Staudinger zum Stadtverordneten gewählt, 1918 bis 1921 war er gar Vorsteher der Stadtverordnetenversammlung. ${ }^{12}$ Anlässlich seines 60. Geburtstages am 2. Dezember 1927 schrieb ihm der Löbauer Stadtrat u. a.: „In den Jahren 1919-1921 haben Sie dem Stadtverordneten-Kollegium als Vorsteher angehört und durch Ihre geschickte und unparteiische Geschäftsführung sich die Achtung und Wertschätzung aller Gruppen erworben."13

Seine Arbeit als Stadtverordneter brachte es mit sich, dass er sich zur Historie der Wasserversorgung der Stadt kundig machte und entsprechende Recherchen im Ratsarchiv anstellte. Plötzlich öffnete sich ihm eine faszinierende Welt historischer Dokumente und Unterlagen. Die Ergebnisse seiner Recherche veröffentlichte er 1915 in der Festschrift des Löbauer Humboldtvereins unter dem Titel „Zu Geschichte der Wasserversorgung der Stadt Löbau“. Es war der Beginn seiner bis an sein Lebensende anhaltenden Leidenschaft - der wissenschaftlichen Erforschung der Löbauer Geschichte und darüber hinaus, der gesamten Oberlausitz.

Bereits 1913 war er Mitarbeiter im Museumsausschuss geworden und betreute in dieser Funktion auch das Stadtmuseum, dessen langjähriger ehrenamtlicher Leiter er dann von 
1928 bis 1943 werden sollte, bis er von den Ämtern im Museum und Archiv zurücktrat. Allerdings übernahm er dann diese Aufgabe auf Bitten der neuen Stadtverwaltung noch einmal von 1945 bis 1951. Zusätzlich war er bereits seit 1922 als ehrenamtlicher Leiter des Ratsarchivs tätig.

Am 9. Oktober 1918 wurde er „wirkliches Mitglied“ der Oberlausitzischen Gesellschaft der Wissenschaften zu Görlitz, ehe diese „den rastlosen und erfolgreichen Forscher in (der) Geschichte der Sechsstadt Löbau“ auf der 236. Hauptversammlung am 29. Mai 1929 zu ihrem Ehrenmitglied ernannte. ${ }^{14}$ Auf der 247. Hauptversammlung am 18. Juni $1941^{15}$ wurde ihm für seine zahlreichen Forschungen und Veröffentlichungen zur Löbauer Geschichte und seine Leistungen für das Löbauer Ratsarchiv die Richard-Jecht-Medaille verliehen. Diese Medaille erhielten neben Staudinger nur fünf weitere Persönlichkeiten der Oberlausitzer Geschichtsforschung.

Anlässlich des 700-jährigen Stadtjubiläums Löbaus am 24. Juni 1921 hielt Otto Staudinger die Festrede unter dem Thema „Die wirtschaftliche Entwicklung der Stadt Löbau seit ihrer Gründung“. ${ }^{16}$ Er veröffentlichte unter dem Titel „Zur Geschichte des Löbauer Gemeindegrundeigentums“ eines seiner Hauptwerke in der Festschrift zum Stadtjubiläum ${ }^{17}$ und im Neuen Lausitzischen Magazin (NLM). ${ }^{18}$

Am 26. August 1921 fand auf Anregung des Oberlehrers Richard Plesky (Pestalozzischule) im Café Babucke auf der Bahnhofstraße 26 die Gründung der „Freien Vereinigung Löbauer Geschichtsfreunde“ statt. Otto Staudinger wurde zum Vorsitzenden gewählt und übte dies Amt bis zum 20. April $1934^{19}$ aus. Am 24. April 1938 ehrte der Oberlausitzer Heimatverband Lusatia (Dachverband der Gebirgs-, Heimatund Humboldtvereine der Oberlausitz) Otto Staudinger mit dem „Lausitzdank“20, und der Humboldt- und Heimatverein Löbau ernannte ihn am 10. Dezember 1940 zu seinem Ehrenmitglied. ${ }^{21}$

Bereits seit dem 1. Februar 1922 gab Otto Staudinger die „Löbauer Heimatblätter“ heraus, die einmal monatlich als vierseitige Beilage des „Sächsischen Postillon“ erschienen. Als die Beilage am 29. September 1939 kriegsbedingt eingestellt wurde, konnten die Herausgeber nach 17 Jahren auf 193 Nummern mit 772 Seiten zurückblicken. Ein Großteil der Texte stammte von Otto Staudinger, der 121 Arbeiten zu verschiedenen Themen der Regional- und Stadtgeschichte und mit unterschiedlichem Umfang beisteuerte. Einige seiner Beiträge erschienen aus besonderem Anlass zusätzlich als
Sonderdrucke, beispielsweise die Geschichte verschiedener Löbauer Innungen. In ungezählten öffentlichen Vorträgen stellte er seine Forschungsergebnisse vor.

Weitere zahlreiche Veröffentlichungen erschienen im Neuen Lausitzischen Magazin, im Sächsischen Postillon und anderen Orten. Mit Kriegsbeginn wurde es immer schwieriger und in der Nachkriegszeit unmöglich, etwas drucken zu lassen. Daher hinterließ Otto Staudinger auch eine Anzahl unveröffentlichter Manuskripte, die später größtenteils dem Stadtarchiv Löbau übergeben wurden. Einige dieser Arbeiten wurden nach 1990 vom Stadtarchiv Löbau für den Druck aufbereitet und veröffentlicht. ${ }^{22}$ Die Stadt Löbau ehrte nach Ende des Zweiten Weltkrieges den besten Kenner ihrer Geschichte, den Kommunalpolitiker, vielfachen Ehrenamtler und Lehrer Prof. Otto Staudinger in sehr würdiger Form. Am 19. Februar 1946 beschloss der Stadtrat die in der Löbauer Neustadt gelegene Hausenstraße in „Otto-Staudinger-Straße“ umzubenennen.

Anlässlich seines 80. Geburtstages am 2. Dezember 1947 beschloss der Stadtrat auf Vorschlag von Bürgermeister Meder am 27. Februar 1948, nach erfolgter vorheriger Zustimmung durch die sowjetische Militärkommandantur, Prof. Otto Staudinger mit der Ehrenbürgerschaft auszuzeichnen. Die Urkunde wurde am 30. April 1948 ausgestellt. ${ }^{23}$

Auch sonst war dieser Geburtstag eine besondere Ehrung für Otto Staudinger. Die „Sächsische Zeitung“ und die „Lausitzer Rundschau“ schrieben Beiträge. Die Wohnung war von Gratulanten (an der Spitze der Bürgermeister und der Stadtverordnetenvorsteher) gefüllt. Im Hungerjahr 1947 waren geschenkte Lebensmittel aber auch Heizmaterial hochwillkommen. Eberhard Staudinger, der Sohn, verfasste darüber einen Bericht für alle in der Familie, die nicht dabei sein konnten. ${ }^{24}$

Am 20. Juli 1951 feierten Otto und Johanna Staudinger bei bester Gesundheit ihre goldene Hochzeit und erstmals seit langem war die ganze Familie aus Ost und West vereint. Ihren jüngsten Sohn und zwei Schwiegersöhne hatten sie schon überlebt, aber es gab inzwischen elf Enkel zur Freude der Großeltern. Kurz nach seinem 85. Geburtstag starb am 7. Dezember 1952 Otto Staudinger in seiner Wohnung Maschinenhausstraße 3, nachdem er sich bereits einige Tage unwohl gefühlt hatte. Sein Grab befindet sich auf dem Löbauer Friedhof. Neben ihm ruht seine Frau Johanna, die ihm 1971 fast 89-jährig nachfolgte. Sie war immer der liebende, kluge und unermüdliche gute Geist für ihren Mann und die große Familie.
14 Zugehörige Urkunden im Familienbesitz.

15 NLM, Bd. 117 (1941), S. 184.

16 Gedenkbuch zum Löbauer Stadtjubiläum. Hrsg. von Dr. Curt Müller. Löbau 1921, S. 108-125.

17 Festschrift 700 Jahre Stadt Löbau, 1221-1921, Löbau 1921.

18 NLM 97 (1921), eine 2. erweiterte Auflage erschien 1942 als Sonderdruck des NLM.

19 Bericht über Entstehung, Entwicklung u. Tätigkeit der „Freien Vereinigung Löbauer Geschichtsfreunde“ von R. Plesky in Löbauer Heimatblätter Nr. 36, Ende Dezember 1925 .

20 Oberlausitzer Heimat, Nr. 8, 17. August 1938, hier wird Otto Staudinger als „bester Kenner der Löbauer Stadtgeschichte" bezeichnet.

21 Urkunde im Familienbesitz.

22 Zum Beispiel: Aus dem Nachlaß Prof. Otto Staudinger, Löbau. (vier Arbeiten) in: Veröffentlichungen des Stadtarchivs Löbau 07/1996.

23 Jörg Zebisch: Otto Staudinger - ein vogtländischer Löbau-Kenner, in: Löbauer Journal, Heft 3 (1994), S. 34-40.

24 Eberhard Staudinger: Bericht vom 80.ten Geburtstag unseres Vaters. Löbau, den 2. Dez. 1947, vier Seiten Schreibmaschine.

\section{Autor}

Gerhard Staudinger Dresden 